\title{
Improved hatchery-rearing techniques for juvenile production of blue swimming crab, Portunus pelagicus (Linnaeus, 1758)
}

\author{
Mhd Ikhwanuddin', Juariah Hafsya Mansor ${ }^{1}$, Abol-Munafi Ambok Bolong ${ }^{1}$ \& \\ Shabdin Mohd Long ${ }^{2}$ \\ ${ }^{1}$ Institute of Tropical Aquaculture, Universiti Malaysia Terengganu, Kuala Terengganu, Terengganu, Malaysia \\ ${ }^{2}$ Faculty of Science and Resource Technology, Universiti Malaysia Sarawak, Kota Samarahan, Sarawak, Malaysia
}

Correspondence: M Ikhwanuddin, Institute of Tropical Aquaculture, Universiti Malaysia Terengganu, 21030 Kuala Terengganu, Terengganu, Malaysia. E-mail: ikhwanuddin@umt.edu.my

\begin{abstract}
The effects of tank colour, larval stocking density, antibiotic administration and water exchange on survival and moulting of blue swimming crab, Portunus pelagicus, were determined. Circular $4-\mathrm{m}^{3}$ experimental larval-rearing tanks were used in triplicate for all treatments. White, dark grey, blue and brown were tested as tank background colours. The stocking densities tested were 10, 20, $40,60,80$ and 100 larvae $\mathrm{L}^{-1}$. The effect of oxytetracycline was estimated by comparing a treatment with oxytetracycline to a treatment without oxytetracycline administration. The daily water exchange rates tested were $0 \%, 25 \%, 50 \%$ and $100 \%$ of the tank volume. In all treatments, the larvae were fed with Artemia nauplii, rotifers and encapsulated Spirulina. The highest percentage survival was observed in the dark-grey tanks when the stocking density of larvae was 20 larvae $\mathrm{L}^{-1}$. No larva reached the juvenile crab size in white tanks. No significant difference in survival was found between treated and non-treated larvae with oxytetracycline when the daily water exchange rate was more than $50 \%$.
\end{abstract}

Keywords: Portunus pelagicus, blue swimming crab, hatchery-rearing techniques, juvenile crab

\section{Introduction}

Blue swimming crab, Portunus pelagicus (Linnaeus, 1758), is distributed throughout the coastal waters of tropical regions of the Indo-west Pacific. The global P. pelagicus capture was $172651 \mathrm{mt}$ in 2007, a small increment when compared with 2004 when the production was $165441 \mathrm{mt}$ (FAO 2007). Portunus pelagicus aquaculture developed very recently, but it is expected to grow into a significant industry. As the aquaculture industry grows, the demand for juvenile crabs will exceed the capacity of the crab fishery. The reliance upon these wild crab juveniles may lead to serious questions about the long-term sustainability of the industry. Hence, the development of a reliable seed-production technology is important for sustainable growth of the industry.

Larvae of the blue swimming crab pass through four zoeal stages (Z1-Z4) and one megalopa (M) stage during development. Moulting for the zoea and megalopa occurs by splitting the exoskeleton at the dorsal boundary between the cephalothorax and the abdomen. The $\mathrm{M}$ stage metamorphoses into the crab stage. According to Arshad, Efrizal, Kamarudin and Saad (2006), the first and second zoeal stages last 3-4 days each, the third and fourth stages last 2-3 days each, and the M stage lasts 3-4 days. The first crab instars lasts from days 15 to 18 after hatching at 28-30 ppt salinity until the next moult.

The larvae culture of P. pelagicus has been studied since the 1970s (Motoh, Pena, Dimaana \& Tampos 1978; Fielder 2004; Ikhwanuddin, Shabdin \& Khairulhayadi 2005; Susanto \& Setyadi 2005; Romano \& Zeng 2006; Soundarapandian, Thamizhazhagan \& John Samuel 2007). However, except for the last two studies, these studies aimed to 